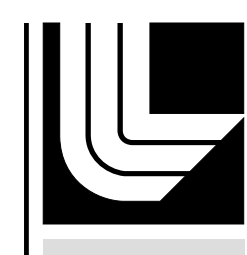

LAW RENCE LIVERMORE N A T IO N A L LABORATORY

\title{
EXPERIMENTS WITH UV LASER TRIGGERED SPARK GAPS IN A STACKED BLUMLEIN SYSTEM
}

W. C. Nunnally, R. Lewis, F. Allen, S. Hawkins, C. Holmes, S. Sampayan, G. Caporaso

June 1, 2005

IEEE International Pulsed Power Conference Monterey, CA, United States June 14, 2005 through June 17, 2005 
This document was prepared as an account of work sponsored by an agency of the United States Government. Neither the United States Government nor the University of California nor any of their employees, makes any warranty, express or implied, or assumes any legal liability or responsibility for the accuracy, completeness, or usefulness of any information, apparatus, product, or process disclosed, or represents that its use would not infringe privately owned rights. Reference herein to any specific commercial product, process, or service by trade name, trademark, manufacturer, or otherwise, does not necessarily constitute or imply its endorsement, recommendation, or favoring by the United States Government or the University of California. The views and opinions of authors expressed herein do not necessarily state or reflect those of the United States Government or the University of California, and shall not be used for advertising or product endorsement purposes. 


\title{
Experiments with UV Laser Triggered Spark Gaps in a Stacked Blumlein System *
}

\author{
W. C. Nunnally ${ }^{++}$, R. Lewis, F. Allen, S. Hawkins, C. Holmes, S. Sampayan, and G. Caporaso \\ Beam Research Group, MS L-645 \\ Lawrence Livermore National Laboratory \\ Livermore, CA 94550, USA
}

\begin{abstract}
This paper discusses switch requirements from basic circuit analysis and the experimental setup, parameters, and results of an experiment to investigate the feasibility of UV laser triggering of up to 40 Blumlein lines in a very compact Stacked Blumlein Line System. In addition, the method of fabricating a very compact SBL transmission lines is presented. Then the behavior of the switch parameters in the stack when closure is initiated with a UV laser pulse is presented. Specifically, the time varying inductance and resistance of the laser initiated gas discharge channel is presented and compared with a circuit model to elucidate the switch performance.
\end{abstract}

\section{STACKED BLUMLEIN LINES}

The Stacked Blumlein Line (SBL) configuration has the potential to provide the most compact pulse power source in that the system volume approaches the dielectric energy storage volume.[1] The critical technology in an SBL system is the high power, stage switch that is required by each Blumlein Line in the stack, as illustrated in Fig. 1. The switches must have inductive transition

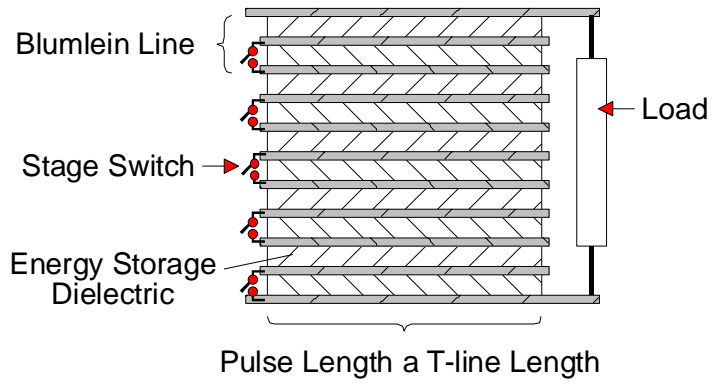

Figure 1. Stacked Blumlein Line System

times and switch resistive phase times that are much less than the desired output pulse width and the closure time must be controllable with a spread much less than the desired output pulse width. The voltage multiplication provided by stacking $\mathrm{N}$ Blumlein lines requires controlling $\mathrm{N}$ switches, in the ideal case, simultaneously.
Closure time variations that are only a small fraction of the designed output pulse duration result in a reduced output voltage, increased risetime, and an increase in the load pulse duration. High voltage gas switches or gas spark gaps and linear rail gap switches have been initiated using pulsed UV lasers with picosecond jitter.

\section{ULTRAVIOLET LASER SPARK GAP TRIGGERING}

Short pulse ultra-violet (UV) laser pulses have been used to initiate the closure of many conducting filaments or arcs in linear rail gap switches [2],[3],[4] The previous work employed a UV laser pulse injected parallel to a rail electrode to produce multiple parallel arc discharges as illustrated in Fig. 2. In this work, a short (10-20 ns) UV

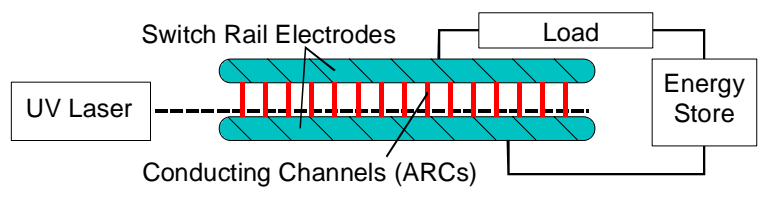

Figure 2. UV Laser Initiation of Parallel Conducting Channels in a Rail Gas Switch

(266 nm) laser pulse was injected along a path such that the laser pulse could initiate the closure of multiple spark gaps, one at each level of the Blumlein Stack as illustrated in Fig. 3. The arrangement of the switch electrodes and the UV laser beam path in the Blumlein Line stack is

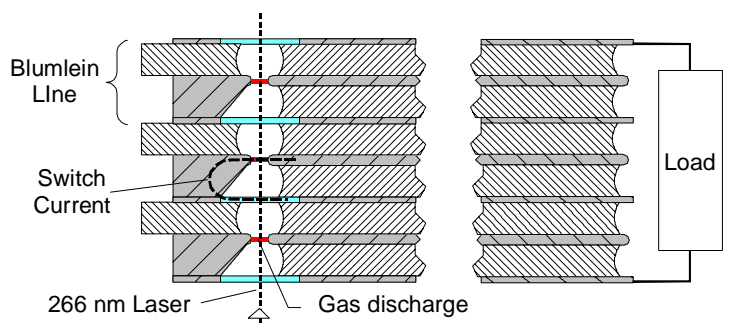

Figure 3. UV Laser Initiation of Series Stacked Blumlein Line Switches

complicated by the compact nature of the dielectric -

\footnotetext{
* This work performed under the auspices of the U.S. Department of Energy by the University of California, Lawrence Livermore National Laboratory under contract No. W-7405-Eng-48.

++ On Leave from the University of Missouri - Columbia, Columbia, MO 65211
} 
conductor stack. For example, in Fig. 3, the gap between two conductors that form the switch for a single Blumlein must be less than the physical separation between those conductors and the Blumlein line conductors above and below each switch. This requires that the dielectric strength of the switching medium, gas in this case, and the dielectric surfaces to be greater than or equal to that of the bulk dielectric. Thus, the switch electrode - laser beam path configuration of Fig. 4 was employed in this work. The geometry of the switch conductors also determines the switch inductance and thus the circuit limit on load pulse risetime. Note that the objective of the experiments described was to investigate the feasibility and limits of UV laser triggering of multiple spark gaps in the very compact SBL configuration.

\section{COMPACT SBL FABRICATION}

The methods and materials used in the UV laser switched SBL affect the performance requirements of the

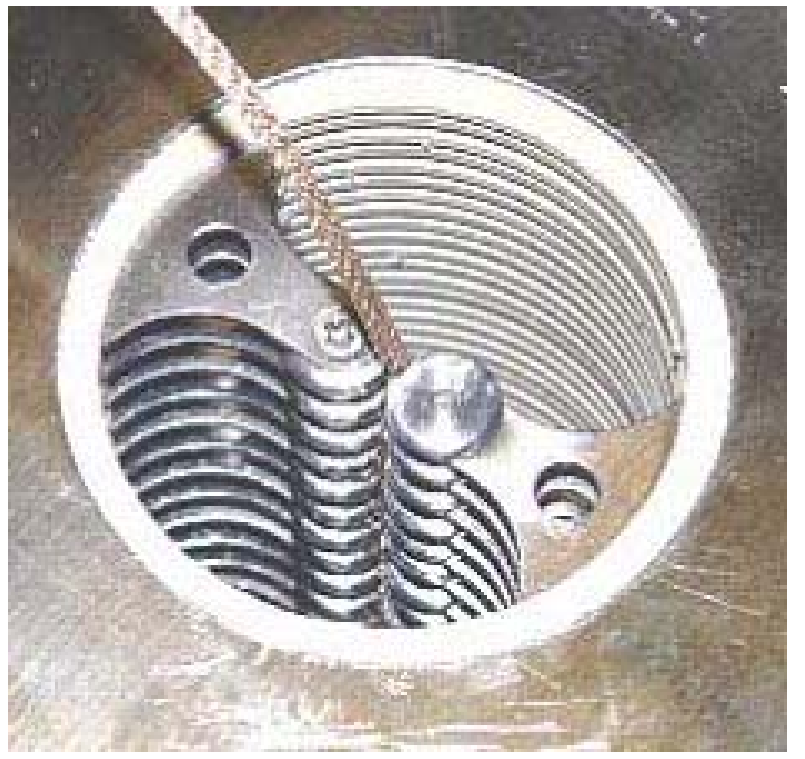

Figure 4. UV Triggered 20 Stack, SBL Switch Electrode Geometry

switch. The dielectric strength of the insulating materials in the transmission lines determines the conductor spacing and thus the spacing allowable for the switch electrodes. In the SBL systems discussed, the conductor spacing was in the range of $0.05-0.1$ inch and the charge voltage in the range of $20-30 \mathrm{kV}$. The nominal switch electrode spacing was set at $2 \mathrm{~mm}$.

The dielectric permittivity in concert with the width of the transmission line conductors, illustrated in Fig. 5, determine the transmission line impedance. The transmission line impedance and the switch conductor inductance then determine the circuit inductive risetime limit. In this work, the conductor thickness was set at 0.032 inch. The most difficult high voltage design problem is related to the elimination (minimization) of surface tracking during the charging process. The

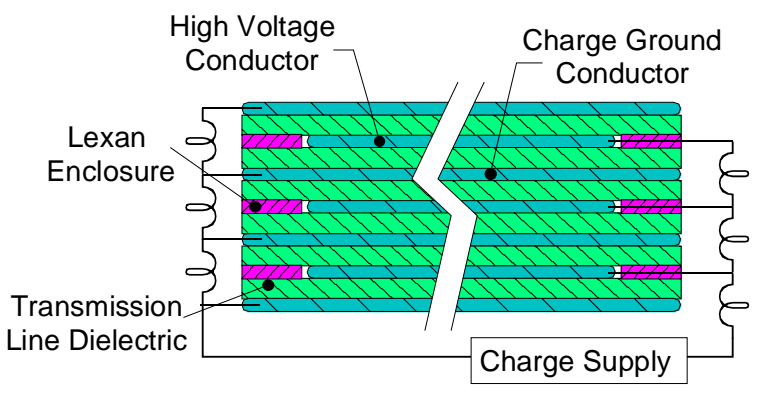

Figure 5. SBL Fabrication cross section

charge voltage in cross section of the SBL, in which alternating layers of conductor and insulator are stacked, was addressed by enclosing the high voltage electrode and the ground structure with dielectric. In this work, adhesives and die cut dielectric materials were bonded in a lay up process that resulted in the system shown in Fig. 6.

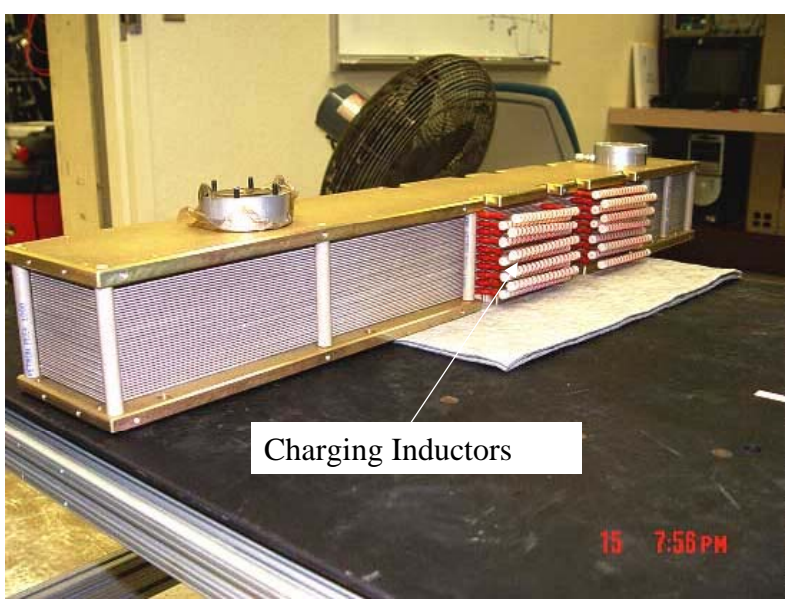

Figure 6. Mod 420 Stack, SBL system

In order to increase the charging voltage to provide a reasonable energy density in the transmission line dielectrics and at the same time reduce the switch electrode separation, the switches were enclosed in a high-pressure gas. In SBL mod 2, the entire system was enclosed in a high pressure (50 psig) gas enclosure and the system charged with a DC voltage to $\sim 25 \mathrm{kV}$. In this system, the tolerance requirements on the switch electrode separation and separation tolerance were found to be on the order of $1 \mathrm{mil}$.

In Mod 3 and Mod 4 SBL systems, a method of placing only the switch electrodes in the high pressure region while maintaining a tolerance of 1 mil was developed. Incorporating all the requirements of fabricating an SBL system were conducted in three steps. First, thin (20 mil) conductor layers were deposited on each layer of dielectric in the shape of the transmission line conductor to insure intimate electrical contact with the high permittivity $(\varepsilon=10)$ dielectric. Each layer of dielectric with conductors was voltage tested to $40 \mathrm{kV}$ to eliminate infant mortality failures and defects in the dielectric. 
Next, the main conductors with spark gap electrodes and supports were bonded to the thin conductors on top of the dielectric slabs using conductive $(0.5$ mil) tape. Simultaneously, the high voltage conductors in the Blumleins were bonded to the next layer of dielectric using Lexan containment structures (See Fig. 5) to form the Blumlein stack. Major Top and Bottom conductors were added to form a rigid structure (See Fig. 6). The most critical spark gap spacings and tolerances were then formed using electric discharge machining of the entire assembly in which the spacing was set at $2 \mathrm{~mm}$ and the spacing tolerance between spark gaps was approximately 1 mil. The Mod 2 SBL system is shown in Fig. 7 and the Mod 4 System shown in Fig. 6.

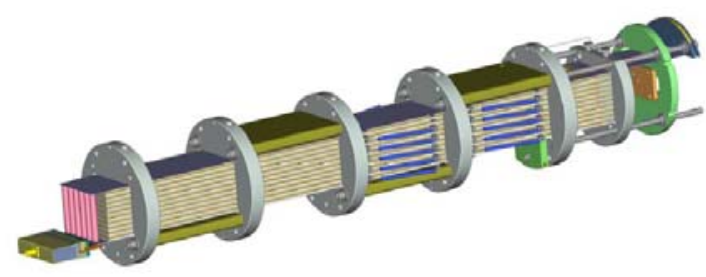

Figure 7. Mod 2, 10 Stack, SBL System

\section{SWITCHING RESULTS}

These experiments were conducted to determine the feasibility of switching multiple Blumlein Lines using a UV laser pulse and to determine the operational parameters and the limitations of the method.

The UV initiation of the spark gap closure is assumed to progress via multi-photon absorption [2] in the gas which produces a sufficient electron density to distort the electric field and initiate a conducting streamer between the electrodes. After the streamer crosses the electrode gap, the high voltage energy available from the circuit then drives current through the streamer to heat the gas and reduce the streamer resistance as it transitions to an arc. After the streamer crosses the electrode gap, the high voltage energy available from the circuit then drives current through the streamer to heat the gas and reduce the arc resistance.

The use of a UV laser has other advantages. UV photons produce additional free electrons at the electrode surfaces that are necessary for streamer initiation. An increase in laser energy can ionize the gas directly to make the region through which the laser passes highly conductive, which more efficiently distorts the local electric field. However, injecting sufficient UV energy to result in gas breakdown with the laser can hinder energy transfer to downstream spark gaps.

A Mod 2SBL was used for the first switching jitter or closure time variation measurements. The optical energy from the arc formation was collected via optical fibers and imaged on the slit of a streak camera to determine simultaneity of switch closure. The specie of gas fill in the spark gaps is a critical factor in initiating gap closure. Three gas mixtures were briefly evaluated: (1) dry air, (2) 80\% Nitrogen, 19 \% Argon, 1 \% Sulfur Hexafluoride (SF6), and (3) dry air with 1\% SF6. The SF6 was added to minimize tracking along dielectric surfaces. Dry air was the superior performer in the quest for synchronous switch closure initiation.

The ten nanosecond streak image is displayed in Figure 8 indicates that switch closure occurs within the optical pulse transit time through the gaps after about 1 ns delay. The streak image of Fig. 8 corresponds with the output in

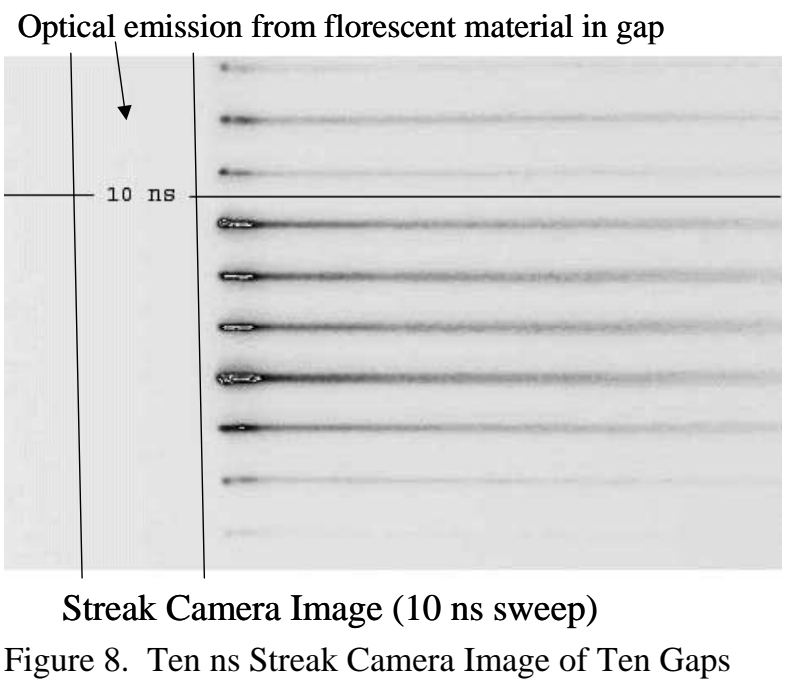

that a synchronous closure of the spark gaps yields a smooth load pulse. Likewise, a larger spread in the streak image is easily seen in an increase in the load pulse width.

\section{MODELING RESULTS}

The amplitude of the experimental load pulse waveforms is much less than initially expected and the rise time is much slower than the inductive time constant limit would permit. Therefore, in order to determine the separate effect of the gas switch resistance during closure and the inductance of the switches, a detailed modeling effort was employed using the MicroCap ${ }^{\mathrm{TM}}$ circuit

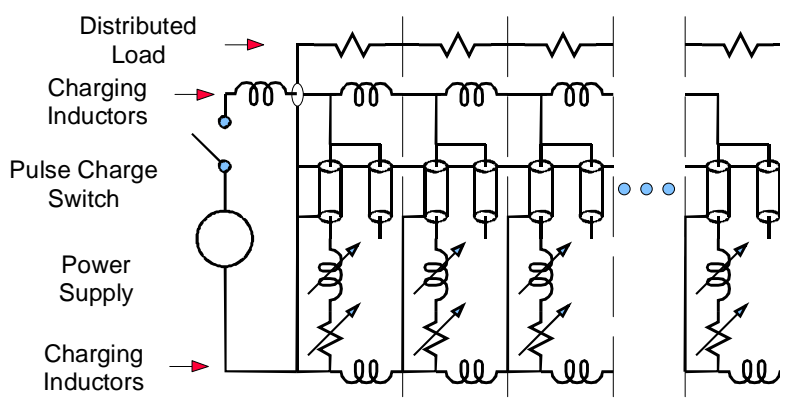

Figure 9. SBL Circuit Model

analysis code. The transmission lines were modeled using lossless T-line models and the resistance and the inductance of each switch modeled as a time varying 
quantity, as illustrated in Fig. 9. The inductance of the switch the Mod 4, SBL switches pictured in Fig. 4 is sufficiently large to make the inductive rise time limit exceed the transmission line pulse width. The switch inductance in Mod 2 SBL systems was a factor of five lower and the transmission line impedance a factor of two larger that than of SBL Mod 4 system. The large difference in inductive time constant for the Mod 2 and Mod 4 systems should have radically altered the pulse rise time. However, the experimental results could only be matched by requiring the switch temporal resistance to decrease in time. The modeling results are compared to the experimental results in Fig. 10 for a Mod 4 SBL

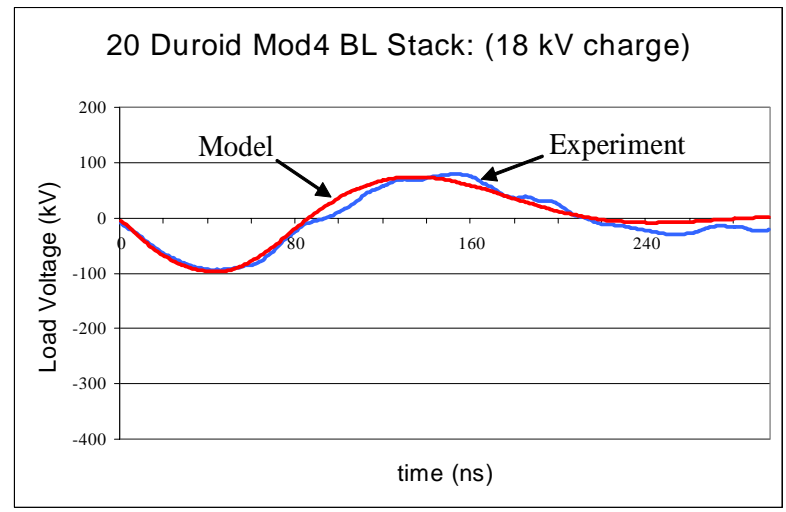

Figure 10. Comparison of Experimental and Model

system. The switch resistance temporal variation required to match the early load pulse behavior is shown in Fig. 11. The switch inductance temporal variation required to match the late time oscillations in the load pulse is shown in Fig. 12. Note that the inductance change required by the model is much too large to be

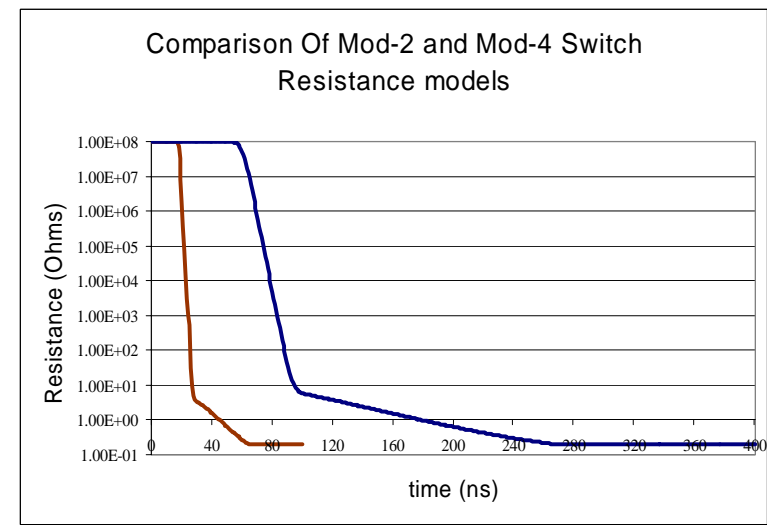

Figure 11. Switch Resistance Temporal Variation

related to the change in the arc diameter change during closure.

Finally, when low resistive phase switch and low inductance switch parameters are used in the model, the potential of the SBL system is compared to the experimental results in Fig. 13.

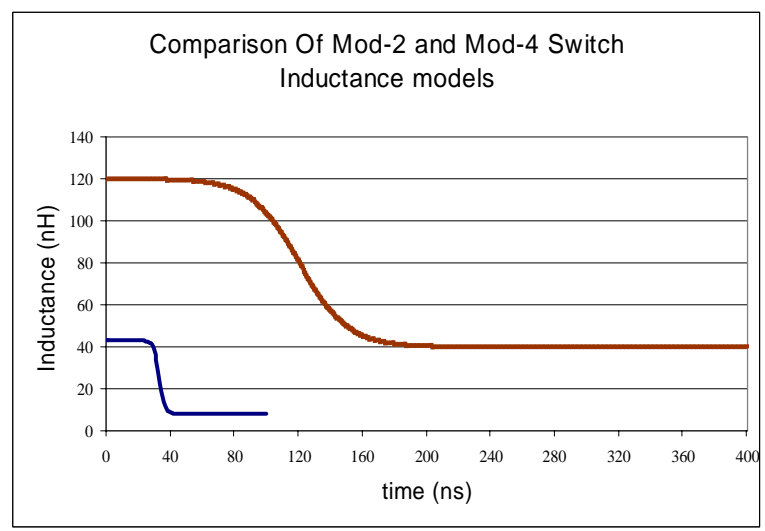

Figure 12. Switch Inductance Temporal Variation

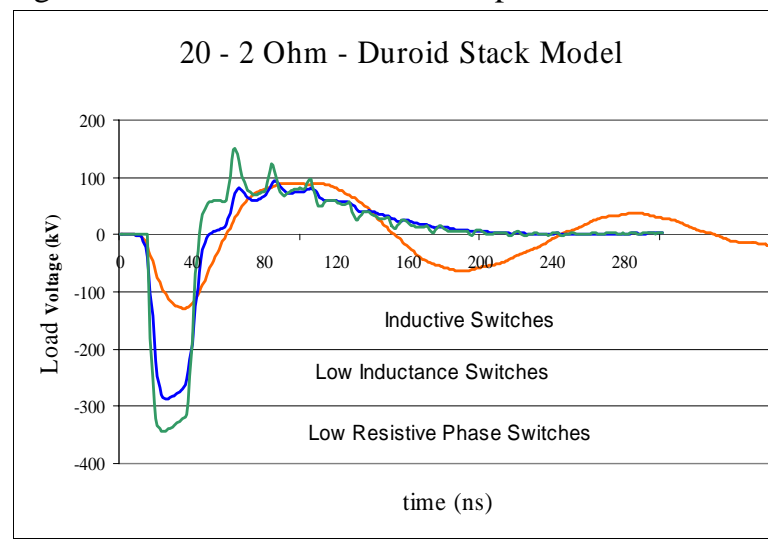

Figure 13. SBL Model with low resistance and inductance parameters

\section{SUMMARY}

The rate of resistance fall in UV laser triggered switches in low (1-2 Ohm) impedance systems and the inductance of a single channel switch are too large to provide fast rise time, high current, high voltage pulses in a high current, high voltage SBL system. Low resistance phase duration, low inductance (multi-channel) switching systems with very low closure temporal variation are required to generate short duration square output pulses. Up to 40 series switches can be closed with sub ns jitter.

\section{REFERENCES}

\footnotetext{
${ }^{1}$ W. C. Nunnally, "Critical Component Requirements for Compact Pulse Power System Architectures,” IEEE Transactions on Plasma Science, August, 2005.

${ }^{2}$ R. S. Taylor and K. E. Leopold, "UV Radiation Triggered rail gap switches,” Rev. Sci. Inst, 55, (1), 1984, pp. 52-63.

${ }^{3}$ A. Endoh and S. Watababe, "Laser Triggering of a 500 kV Rail Gap Switch,” J. Appl. Phys, 59 (10) 15 May 1986, pp. 3561-3565

${ }^{4}$ J.R. Woodworth, C.A Frost, and T.A. Green, "UV Laser Triggering of High Voltage Gas Switches,” J. Appl. Phys, 53(7), July 1982, pp. 4734-4739.
} 